Noname manuscript No.

(will be inserted by the editor)

\title{
MPR-Aware Scheduler for Carrier Aggregation Transmissions in LTE Uplink
}

\author{
Maria A. Lema · Mario Garcia-Lozano . \\ Silvia Ruiz
}

Received: date / Accepted: date

\begin{abstract}
Unlike previous Long Term Evolution (LTE) releases, LTE - Advanced (LTE-A) allows the use of non-contiguous resource allocation in the uplink (UL). This feature leads to increase the spectral efficiency thanks to link performance gains obtained from frequency diversity. At the same time, non-contiguous allocations bring higher peak to average power ratio (PAPR) and so potentially higher intermodulation distortion and adjacent channel leakage-power ratio (ACLR). Power derating has been proposed as a means to avoid this problem and thus user equipments (UEs) must reduce their maximum transmitted power. However, the additional link loss on power limited UEs partially counteracts the gain brought by multi-cluster allocation. In this sense, this paper proposes to include Maximum Power Reduction (MPR) information in opportunistic scheduling decisions. The new scheduling proposal is able to determine whether multi-clustering leads to a net gain in instantaneous throughput or if localized allocation is preferred. The proposed method is compared to other scheduling techniques and it is also analyzed in different inter-site distance scenarios. Results show that considering MPR in opportunistic decisions can lead to overall cell performance improvements.
\end{abstract}

Keywords LTE-A · CA · UL Scheduler · Cell-edge · MPR · Power Control · non-contiguous RA

\section{Introduction}

Long Term Evolution - Advanced (LTE-A) is the 3GPP standard that addresses IMTAdvanced requirements for the new generation of mobile communications. Presented

Maria A. Lema, Mario Garcia-Lozano and Silvia Ruiz Department of Signal Theory and Communications

Universitat Politecnica de Catalunya (UPC), BarcelonaTech

Barcelona, Spain

E-mail: $\{$ maria.lema, mariogarcia, silvia $\}$ tsc.upc.edu 
in Release 10 and 11, this standard aims at fulfilling the market needs of the next decade.

Given the demanding requirements set for the fourth generation (4G), LTE-A had to introduce new capabilities in order to cope with them. Among others, the most innovative ones are: wider bandwidth support, uplink (UL) multiple input multiple output (MIMO), higher order MIMO for the downlink (DL), heterogeneous network support and coordinated multipoint transmission. The specified figures given for spectral efficiency (average and cell edge) for both UL and DL, latency and availability of scalable bandwidth are accomplished and even exceeded the IMT-Advanced requirements [1].

Wider bandwidth support by means of carrier aggregation (CA) improves data rates, load balancing and increases spectrum utilization. All this, by allowing the simultaneous transmission of data in different component carriers (CCs). Other reasons that motivate the use of CA techniques are the spectrum holdings located across several frequency bands and the coexistence of different radio access technologies (RAT). Indeed, multi-RAT CA is a matter of study for Release $12[2,3]$.

The inclusion of CA in the DL is almost immediate as orthogonal frequency division multiplexing (OFDM) already provides flexibility in the resource allocation (RA). However, the use of this technology in the UL is not as direct as in the DL case. Release 8 LTE selected localized single-carrier frequency division multiplexing (SC-FDMA) in which symbols are transmitted serially, thus reducing the signal peak to average power ratio (PAPR). However, due to the new configuration of CCs the single carrier property cannot be maintained since non-contiguous RA must be performed. Therefore, other solutions must be adopted in order to support CA in the $\mathrm{UL}$.

3GPP has agreed to use N x Discrete Fourier Transform - Spread - OFDM (N x DFT-S-OFDM) to cope with the new spectrum configuration. It is a good solution due to its compatibility with LTE as each transport block is independently DFT spread and then mapped over the CCs [4]. Non-contiguous RA is allowed in each CC [5], thus clustered DFT-S-OFDM is used. Separated pieces of spectrum known as clusters are allocated to users, with the inconvenience of increasing the PAPR [6]. Nevertheless, this solution provides less PAPR than pure OFDM and it constitutes a good compromise between SC-FDMA and OFDM [7]. Physical resource blocks (PRBs) can be allocated with more flexibility, which yields to more frequency diversity gain.

Usually, when intra-band contiguous CCs are aggregated, the transmitter uses the same power amplifier (PA) for all carriers. The PAs used in radio transmitters have non-linear characteristics, which cause significant distortion in the signals whose instantaneous power fluctuations come too close to saturation level.

Maximum Power Reduction (MPR) is introduced when multi cluster and CA transmissions are carried out [8]. With this, non-linearities on the PA are contained and the spectrum emission mask is respected. Reducing the maximum available power at the UE side ensures a lower working point of the PA, which guarantees linearity. Of course, this power de-rating can severely affect the whole performance, where the link budget is the worst and user equipments (UEs) are likely to be power limited. Further reductions on power may reduce cell edge performance. Users located at the 
cell limit already transmit at maximum power levels as they experience the worst channel conditions.

Given this, MPR clearly impacts the UL performance at a system level. Not all UEs are valid candidates for adopting CA or multi cluster transmission because their throughput could be reduced if compared to the a localized allocation. However, as previously indicated, non contiguous RA brings an intrinsic frequency diversity gain that, in some cases, could well overcome the extra losses due to MPR. Following this thought, each UE should be independently assessed to verify whether a CA transmission would imply a net gain in throughput. Otherwise, Release 8 conditions (use of SC-FDMA) ought to be maintained to not impair their quality of service.

This work presents a novel scheduler that considers MPR information opportunistically in the scheduling decisions. Initially, all users are assumed to be appropriate for CA transmission. Next, in order to evaluate if a non contiguous RA is suitable, their throughput is estimated. This is done based on the information from past sounding reference signals (SRSs) received by the eNode-B (eNB) from the UE. The MPR to be eventually applied depends on several parameters such as the number of CCs and assigned bandwidth. The scheduler will compute the best combination, so that its final allocation decision maximizes the UE throughput.

The rest of the paper is organized as follows: Section II collects all the related references and prior work done. Section III introduces the theoretical background of MC transmission in CA, and in section IV the novel scheduling algorithm is presented. The simulation framework is explained in section $\mathrm{V}$, followed by the results in section VI. To end with this contribution, conclusions are drawn.

\section{Related Literature}

Prior art related to this topic can be divided into two different research lines. The first one gathers the works that investigate scheduling in the CA and non contiguous context, topics like fairness and coverage maximization are of major interest. The second research line refers to the $\mathrm{CC}$ selection process, a new radio resource management operation in charge of deciding which UEs are suitable of transmitting simultaneously in more than one carrier. This line focuses on the system capacity improvement by allocating the number of CCs that maximizes the overall performance.

Regarding the first group of contributions, works in [9] and [10] present two approaches of scheduling. The first one, investigates the performance of schedulers operating in a per-CC basis, so information about throughput in other $\mathrm{CCs}$ remains unknown and proportional fairness is impaired. The second work evaluates the gains of cross-carrier scheduling and sharing past throughput information from all CCs. Results of this contribution do not present such a gain in terms of average throughput, however it presents an important increase of coverage gain. The same results are obtained analytically in [11], which proves that the cross CC proportional fair scheduler maximizes the network utility. It also improves fairness by increasing the scheduler priority of users that cannot access all CCs. Authors in [12] also deal with independent versus cross-CC scheduling. After quantifying the benefits of the latter in different scenarios, they observe that users with diverse performance in available 
CCs are not uniformly scheduled among them. Hence, they propose a Layer-3 management scheme that configures a particular number (and choice) of CCs to specific users, thus obtaining the same results as joint scheduling but with less complexity.

All these scheduling discussions are centered in the DL, in which CA is not exploited in the same manner as in the UL. Authors in [13] tackle the scheduling problem in the UL from an energy efficiency point of view. A dynamic scheduler is proposed, in which each CC can collaborate with the others to assure that the total capacity of all CCs is fully utilized; two different cooperation approaches are presented. Results show that the same average throughput as in joint scheduling can be obtained with an efficient reduction in power consumption. Also, the balancing capacity is improved. All UEs can use multiple CCs simultaneously and it does not consider power de-rating when simultaneous transmission across more than one CC occurs. Work in [14] studies the system performance of clustered DFT-S-OFDM. No $\mathrm{CA}$ is considered but sparse allocation of PRBs along one carrier is. Due to frequency selective gain and scheduling flexibility uplink transmissions improve. Authors propose two methods of MPR, one with a fixed value and another one in which the power de-rating varies depending on the bandwidth allocated, solution that is quite close to the conclusions by $3 \mathrm{GPP}$ in [8].

References tackling the CC selection can be easily found in the DL with interband CA deployments, where carriers have different radio electric propagation conditions. In [15] it is proposed a geometry factor based algorithm. Users far from the eNB transmit in the carrier that provides larger coverage, and the rest are allocated following load balancing policies. Results show that the CC selection process can improve coverage performance at the expense of marginal average throughput loss. Another related work is [16] where the decisions are based on the Reference Signal Received Power (RSRP) and also the average rate of past transmissions, which increases fairness. Authors in [17] develop a CC selection process based on both: propagation conditions and load balancing policies. Results show that the system performance is improved as more dimensions are considered. Linked to the previous, [18] introduces a per-UE weight variable per CC based on the user path-loss and the occupancy of the CC. With this, both problems are tackled: radio electric propagation and load balancing. All these works consider that there are UEs who are not capable of transmitting in more than one carrier, and the scheduler considers these as pure Release 8 UEs. However, in the DL the main reasons to deprive one UE from being allocated in more than one carrier is congestion and coverage; whereas in the UL there are more variables to evaluate.

UL CC selection must be different than the DL techniques, given that the throughput is ruled by the UE availability of power. Extending bandwidth to users not always results in a performance gain. In maximum power transmission situations an increased bandwidth may jeopardize the UE capacity. Gains brought by adopting CA in the UL are analyzed in [14]. A power back-off factor is introduced to limit the power among the different CCs, however no MPR is reckoned in this study. No gain is perceived in the cell-edge coverage because power limited LTE-A users are treated as Release 8 users, allocating contiguous PRBs in one CC. Reference [19] is probably the most representative and relevant work dealing with UL CA selection of UEs. Here, users are allocated one or multiple CCs based on the path-loss. A threshold is 
Table 1 Summary of References. N.A: Not Applicable

\begin{tabular}{ccccc}
\hline \multicolumn{5}{c}{ Literature review summary } \\
\hline Ref \# & Link & CA & MPR & Cell-edge \\
\hline$[9,10,11]$ & DL & $\checkmark$ & N.A & $\boldsymbol{x}$ \\
{$[12]$} & DL & $\checkmark$ & N.A & $\boldsymbol{x}$ \\
{$[15]$} & DL & $\checkmark$ & N.A & $\checkmark$ \\
{$[16]$} & DL & $\checkmark$ & N.A & $\checkmark$ \\
{$[17]$} & DL & $\checkmark$ & N.A & $\checkmark$ \\
{$[18]$} & DL & $\checkmark$ & N.A & $\checkmark$ \\
{$[13]$} & UL & $\checkmark$ & $\mathbf{x}$ & $\mathbf{x}$ \\
{$[14]$} & UL & $\mathbf{x}$ & $\checkmark$ & $\checkmark$ \\
{$[20]$} & UL & $\checkmark$ & $\checkmark$ & $\mathbf{x}$ \\
{$[22]$} & UL & $\checkmark$ & $\mathbf{x}$ & $\mathbf{x}$ \\
{$[19]$} & UL & $\checkmark$ & $\mathbf{x}$ & $\mathbf{x}$ \\
{$[21]$} & UL & $\checkmark$ & $\mathbf{x}$ & $\checkmark$ \\
\hline
\end{tabular}

calculated to distinguish between power limited and non-power limited LTE-A users. This value must be fine tuned to assure the maximization of both, cell-edge and average throughput. Besides, the optimum value depends on the inter site distance (ISD) and might be quite different in rural or high dense urban environments. This study does not consider multi-cluster transmission in one CC, and also MPR is set to be the same value regardless the allocated bandwidth. Moreover in [20] the same pathloss threshold is applied with multi-user multiple input multiple output (MU-MIMO) techniques. This work considers the MPR and also multi clustering in one CC. However, access to CA and multi cluster transmission is limited to those users that succeed a given path-loss threshold. Meaning that both CA and non-contiguous RA is limited to the inner UEs. In the same line there is [21], in which inter-band CA is considered. Here, authors claim that UEs in the cell edge have less CCs with good channel quality than those of the cell centre. If both type of users have the same traffic requirements then the assignment of equal number of CCs may result unfair. So, users with similar channel conditions are grouped, and the cell edge groups are prioritized in the $\mathrm{CC}$ assignment. This cell edge oriented CC selection algorithm is compared to round robin and opportunistic CC assignments showing improvements in the cell edge.

Table 1 summarizes the highlights of the references described. In the majority of the UL works cell edge UEs are not considered for spectrum aggregation, which are basically treated as Release 8 UEs and the new capabilities brought by LTE-A are not exploited. Power de-rating is not included in most of the UL works, and realistic CSI with the use of SRSs is never mentioned.

The work presented in this paper allows CA and non-contiguous transmission in one CC to all UEs in the scenario. One single packet scheduler is considered and the entire resource pool is available. The novel scheduler considers power de-rating if necessary, and allocates the resources that maximize the UE capacity. All users transmit the same bandwidth in a contiguous or non-contiguous manner depending on the 


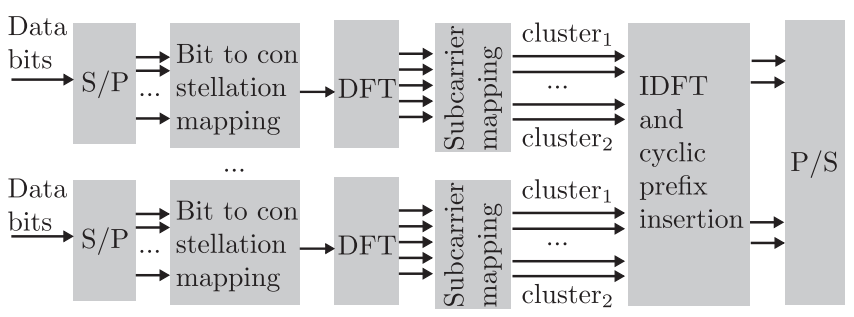

Fig. 1 NxDFT-D-OFDM signal generation

scheduler decisions. The methodology that has been followed to test this novel strategy is system level simulations with a comparative study against previous proposals. This has been done in a detailed manner, considering channel state information (CSI) realistically through the allocation of SRS (pre-scheduling process).

\section{Multi-cluster Transmission in CA}

In previous releases, SC-FDMA was selected to be the multiple access technique adequate for the UL. By allocating UEs a set of contiguous sub-carriers, signals achieved very low PAPR compared to the OFDMA solution. This is a good option as it facilitates the use of efficient power amplifiers in the devices. However, the contiguous allocation impairs scheduling flexibility and reduces frequency diversity.

Given the new spectrum configuration, allowing the simultaneous transmission of data in different frequency carriers, results in a higher PAPR due to non-linearities of the power amplifier, as the single carrier property can no longer be maintained. The solution adopted is NxDFT-S-OFDM due to its good compatibility with previous releases of LTE [23]. Non-contiguous RA within one CC is done grouping the physical resource blocks (PRBs) in clusters of contiguous sub-carriers. So, each CC is independently DFT spread, and each transport block is built of non consecutive clusters of PRBs, figure 1 shows the new signal generation schematic. In the context of LTE-A the maximum number of allocable clusters is two per $\mathrm{CC}$, and the number of PRBs per cluster depends on the system bandwidth [8].

The use of parallel transmissions still impacts the spectrum mask and there can be out of band emissions due to the intermodulation products of the parallel signals. This drives the need for maximum power reduction which depends on the signal generation, as seen in figure 2, where variables that impact the PAPR of the signal are shown.

Several authors have worked on obtaining an accurate characterization of the MPR given the spectrum configuration of CA. Authors in [25] concluded that a single metric can be used to determine the required MPR when two CCs are aggregated. This metric is the ratio between the total allocated bandwidth and the entire available bandwidth. Moreover, authors in [24] present an extension of the previous study with further simulations and propose a more accurate estimation of the MPR to be applied. Given these studies, the 3GPP included a MPR mask equation for multi cluster transmission in both multi-CC and single-CC transmission. For intra-band contiguous $\mathrm{CA}$ 


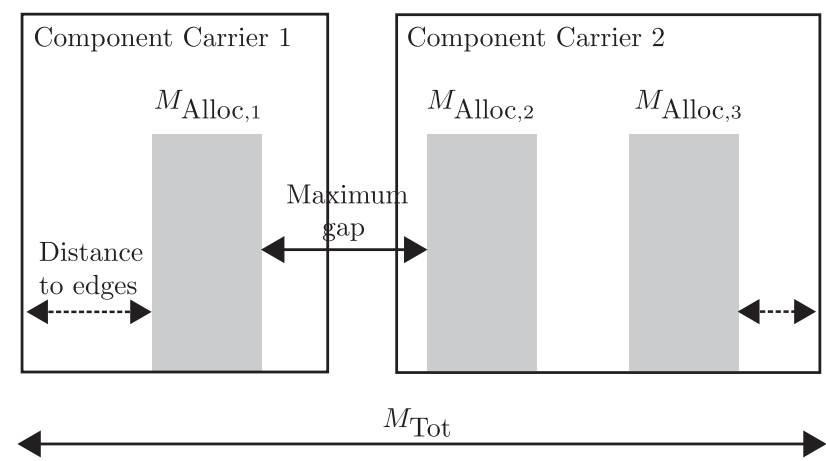

Fig. 2 Variables of the aggregated signal that affect in the PAPR, [24]

the formula agreed is:

$$
\pi_{A}^{\mathrm{CA}}= \begin{cases}8.2 \cdot A & \text { if } 0<A<0.025 \\ 9.2-40 \cdot A & \text { if } 0.025 \leq A<0.05 \\ 8-16 \cdot A & \text { if } 0.05 \leq A<0.25 \\ 4.83-3.33 \cdot A & \text { if } 0.25 \leq A<0.4 \\ 3.83-0.83 & \text { if } 0.4 \leq A \leq 1\end{cases}
$$

As for SC transmissions the MPR for multi clustered allocations is given by:

$$
\pi_{A}^{\mathrm{SC}}= \begin{cases}8-10.12 \cdot A & \text { if } 0<A \leq 0.33 \\ 5.67-3.07 \cdot A & \text { if } 0.33<A \leq 0.77 \\ 3.31 & \text { if } 0.77<A \leq 1\end{cases}
$$

In both equations, $A=M_{\text {Alloc }} / M_{\text {Tot }}$ is the ratio between the allocated PRBs and total PRBs in the system bandwidth. In the case of CA, the system bandwidth corresponds to the total aggregated one. This mask applies the maximum reduction of power for narrow allocated bandwidths, which confirms what was concluded in [26].

\section{UE power setting and MPR}

The open loop power control (OLPC) in LTE is the basic mechanism to compensate for slow varying pathloss conditions. The UE adjusts its transmission power $P$ as:

$$
P=\min \left(P_{\max }, P_{0}+10 \log M_{\text {Alloc }}+\alpha \cdot L\right)
$$

Where:

- $P_{\max }$ maximum allowed power, depending on the UE power class [8].

- $P_{0}$ controls the average received power spectral density.

- $\alpha$ is the path loss compensation factor. It is tuned to allow partial path loss compensation, which combined with an adequate $P_{0}$ value allows significant UE throughput improvements [27, 28]. 
- $L$ is the downlink path-loss measured at the UE.

Given equation 3 , increasing the transmission bandwidth $M_{\text {Alloc }}$ may not be beneficial for all UEs, as the total transmitted power depends on the allocated bandwidth. This is particularly true for cell edge UEs, which are much more likely to transmit at maximum power levels. Unless $P_{\max }$ is reached, power per PRB is constant regardless the allocated bandwidth, then the power spectral density drops as the number of PRBs grows. However, with the inclusion of non contiguous RA, the maximum power is reduced, and the power per PRB can severely decrease. The total transmitted power in the physical uplink shared channel (PUSCH) of a CA system is:

$$
P_{\mathrm{PUSCH}}^{\mathrm{CA}}=\min \left(P_{\max }^{\mathrm{MC}}-\pi_{A}^{\mathrm{CA}}, 10 \log P^{\prime}\right)
$$

Where $P^{\prime}$ is the final sum of the $N$ aggregated CCs:

$$
P^{\prime}=\sum_{i \in N} P_{0_{i}} M_{\text {Alloc }_{i}} \alpha_{i} L_{i}
$$

Equation 3 is used to determine the transmission power on each $\mathrm{CC}$, named $\mathrm{CC}$ specific power control. The need of specific power setting arises as there might be aggregation scenarios with potentially different propagation conditions or interference conditions. This means that different OLPC parameters can be selected, and that a UE can transmit using different transmission power and power spectral density on the multiple CCs. If the total transmission power on all CCs exceeds the maximum UE power capabilities, the UE must decide how to reduce the transmission power and determine this power reduction for each CC [29].

If the eNB does not know how close the UE is of its maximum transmit power, it can allocate resources for which the UE can not respond efficiently due to a lack of power. Power headroom reports (PHRs) indicate how much transmission power is left for a UE with respect to the power being used by the current transmission. In Release 10 the CC specific maximum power limit is included in the PHR [30, 31]. Both PHR and CSI provide the eNB with sufficient information to allocate more efficiently. The CSI is obtained through SRSs which is a reference signal for the eNB to learn about the channel quality for each subsection of frequency region.

Note that SRS power is also adjusted with the same OLPC algorithm (Equation 3 ), where $M_{\text {Alloc }}$ corresponds to the SRS bandwidth allocated, $M_{\text {SRS }}$. Sounding and data might have different allocated bandwidths, but the power spectral density (PSD) $\delta$ measured at the PRB level is kept as long as power levels are below $P_{\max }-\pi_{A}$.

$$
\delta=\min \left(P_{\max }-\pi_{A}-10 \log M_{\text {Alloc }}, P_{0}+\alpha \cdot L\right)
$$

On the contrary, if $\delta_{\mathrm{SRS}} \neq \delta_{\mathrm{PUSCH}}$ the CSI brought by the SRS can be significantly different from the PUSCH.

Based on this, it is necessary to develop a scheduling entity that considers MPR opportunistically in scheduling decisions, and allocate separate clusters or carriers or decide a localized single carrier transmission only if this results in a throughput improvement. 


\section{Sytem Model}

Consider a macro cell network with a set of $\mathscr{J}$ eNBs, each one has a total number $\mathscr{I}$ of associated users. Each eNB employs $\mathscr{L}$ CCs in the same frequency band with the exact same bandwidth. Each CC is comprised of $\mathscr{R}$ PRBs which are allocated to users each time transmission interval (TTI).

Frequency selective scheduling is possible in the UL due to SRSs, which are transmitted periodically or occasionally, depending on the UE needs. SRSs are sent in the last SC-FDMA symbol, in which all the PRBs are reserved for the same purpose. UEs at the cell edge typically transmit hopped SRSs to have more reliable measurements due to power limitations. Simultaneous SRSs in different CCs are allowed, however power scaling may be necessary; in order to avoid it, this work alternates SRSs of different CCs in different TTIs .

The eNB allocates a set of $M_{\mathrm{SRS}}$ contiguous PRBs to be sounded and obtain a CSI measurement. The signal received per PRB $r$ at the eNB side in its $l$ th component carrier and one round trip time (RTT) later is denoted as $S_{l, r}^{\mathrm{UL}}(i, j)$. For the sake of simplicity subindex $l$ has been omitted in all equations

$$
S_{r}^{\mathrm{UL}}(i, j)=P_{\mathrm{SRS}} h_{r}(i, j) d(i, j)^{-\alpha} 10^{\frac{\chi}{10}}
$$

Where $P_{\mathrm{SRS}}$ is the transmitted power from equation $3 ; h_{r}(i, j)$ is the Rayleigh fading at PRB level; $d(i, j)$ is the distance from user $i$ to the eNB $j$ and $\alpha$ is the path loss exponent; $\chi$ is a Normal random variable with zero mean and standard deviation $\sigma$.

With this, the eNB can estimate the user's $i$ CSI at PRB $r$ in carrier $l$ as:

$$
\gamma_{r}^{\mathrm{SRS}}(i, j)=\frac{S_{r}^{\mathrm{UL}}(i, j) d(i, j)}{I_{r}(i, j)+\sigma_{n}^{2}}
$$

where $\sigma_{n}^{2}$ is the noise power of the additive white Gaussian noise and $I_{l, r}$ is the total aggregated inter-cell interference perceived in PRB $r$ and is modeled as:

$$
I_{r}(i, j)=\sum_{n \in N} P_{\mathrm{SRS}, n} h_{r}(i, j) d(i, j) d(n, j)^{-\alpha} 10^{\frac{\chi}{10}}
$$

where $N$ is the set of interfering uplink users associated to the neighbouring cells.

Following this sounding process along the entire $\mathscr{R}$ lets to obtain a single value of $\gamma_{r}^{\mathrm{SRS}}(i, j)$ for every PRB. This information is used by the scheduling entity to perform spectrally efficient decisions and also for the link adaptation to assign the most appropriate MCS.

The scheduler's main task is to allocate $M_{\mathrm{PUSCH}}$ in order to maximize throughput while maintaining a proportional fairness in the coverage area. Based on the average $\overline{\gamma^{\mathrm{SRS}}(i, j)}$ over the allocated PRBs, the eNB signals the UL scheduling grant.

The received PUSCH signal one RTT later from the allocation is:

$$
S^{\mathrm{UL}}(i, j)=P_{\mathrm{PUSCH}} h(i, j) d(i, j)^{-\alpha} 10^{\frac{\chi}{10}}
$$


where $P_{\mathrm{PUSCH}}$ is obtained from the OLPC algorithm with the corresponding MPR reduction in case of multi cluster allocation (Equations 3 and 4). The resulting SINR is:

$$
\gamma^{\mathrm{PUSCH}}(i, j)=\frac{S^{\mathrm{UL}}(i, j)}{\overline{I(i, j)}+\sigma^{2}}
$$

where $\overline{I(i, j)}$ is the average interference perceived in all $M_{\mathrm{PUSCH}}$.

From equations 8 and 11 it is inferred that a difference in both may arise if the user transmits at maximum power levels. This misalignment is mainly due to $\delta_{\mathrm{SRS}} \neq$ $\delta_{\mathrm{PUSCH}}$, and it is quantified as $\Delta P$ :

$$
\Delta P=P_{\mathrm{SRS}}-P_{\mathrm{max}}+\pi_{A}+10 \log \frac{M_{\mathrm{PUSCH}}}{M_{\mathrm{SRS}}}
$$

\section{Multi-cluster Scheduler for Joint CC Resource Allocation}

Based on the explanation done in previous sections, dealing with non contiguous transmissions may imply a loss in the UL performance which can be counteracted by the intrinsic frequency selective gain.

The proposal of this work is extending scheduling information so that algorithms account for MPR information, power headroom reports from the user and anticipating possible SINR imbalances between information from SRS and their allocated power and the eventual power used in scheduled PUSCH resources. The main goal of this new functionality is to assess if non contiguous allocations lead to a net gain in instantaneous throughput when compared to a localized single carrier treatment. In other words, it decides which UEs are eligible for uplink CA. No extra signalling is needed to include this new operation.

Our general allocation model is a hybrid automatic repeat request (HARQ) aware scheduler based on proportional fair decisions that has been added the capability of allocating separated clusters of both CCs. The scheduler is divided in two parts as presented in [32]. First a time domain (TD) scheduler followed by a frequency domain (FD) one. The new operation features are included in Algorithm 1 which is explained in detail in the following paragraphs.

The TD scheduler is in charge of sorting users following a proportional fair policy and generating the final reduced set of uplink users allowed to be served in the current TTI. The procedure is HARQ aware and so the subset of users $\mathscr{H}$ with pending re-transmissions is included in the group. Then, the scheduler completes the list following the prioritization metric $\tau$ computed for every user $i$ that sent a scheduling request $(0<i<I)$ :

$$
\tau(i)=\frac{R_{\mathrm{b}}^{\mathrm{G}}(i)}{\overline{R_{\mathrm{b}}}(i)}
$$

where $R_{\mathrm{b}}^{\mathrm{G}}(i)$ is the bitrate to be granted for the current service of $i$ and $\overline{R_{\mathrm{b}}}(i)$ is its past average throughput. 


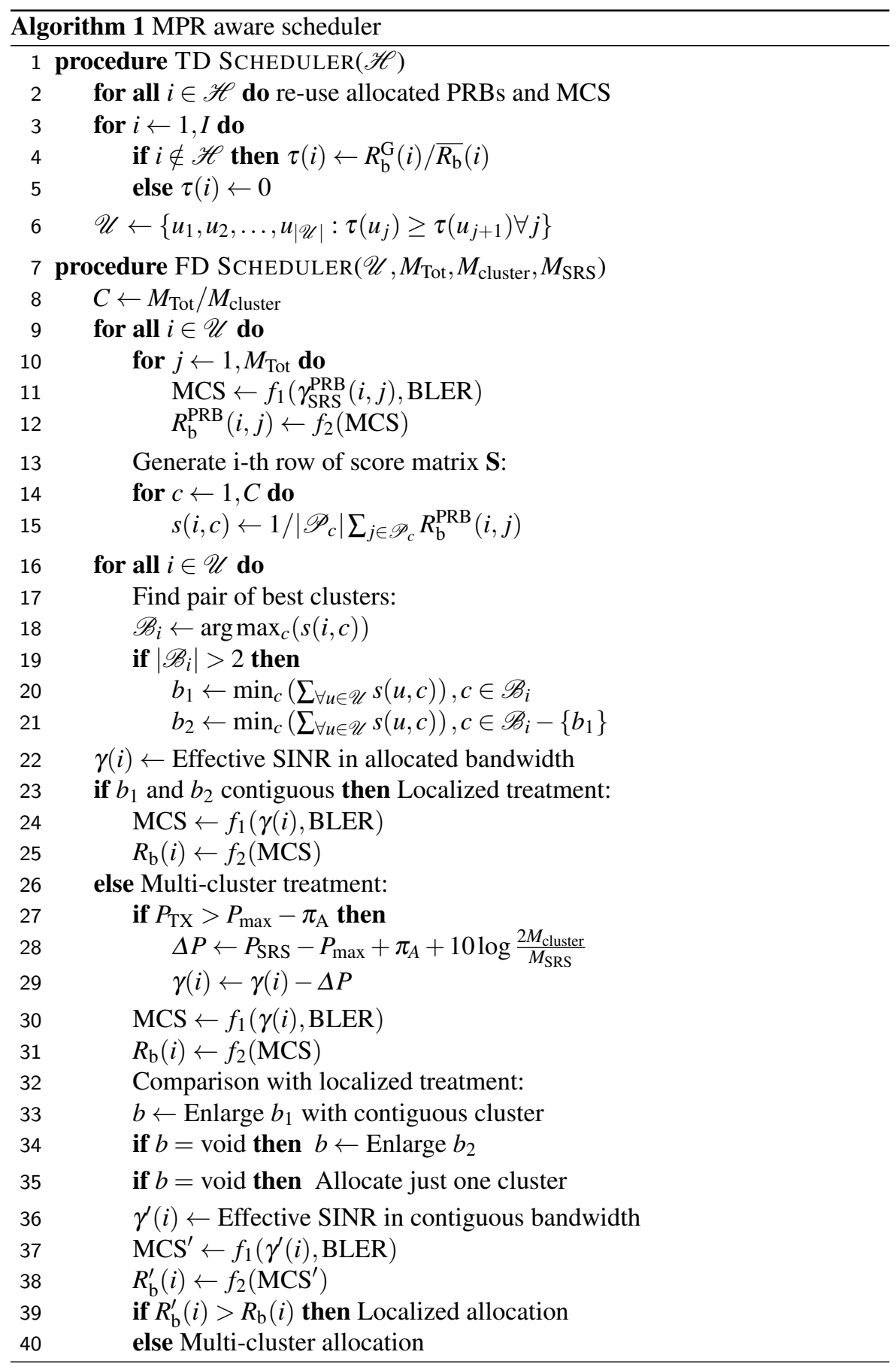


From the final sorted list, a subset of users is chosen to be served in the current TTI.

$$
\mathscr{U}=\left\{u_{1}, u_{2}, \ldots, u_{|\mathscr{U}|}: \tau\left(u_{j}\right) \geqslant \tau\left(u_{j+1}\right) \forall j\right\}
$$

The system bandwidth is divided into $C$ clusters of $M_{\text {cluster }}$ PRBs each one, therefore the size of $\mathscr{U}$ is given by:

$$
|\mathscr{U}|=\max \left(\frac{M_{\text {Tot }}}{2 M_{\text {cluster }}}-|\mathscr{H}|, 0\right)=\max \left(\frac{C}{2}-|\mathscr{H}|, 0\right)
$$

The FD scheduler allocates PRBs to UEs in $\mathscr{U}$ aiming at maximizing the spectral efficiency. On the other hand, synchronous non-adaptive HARQ is considered, and so re-transmissions re-use the same allocation bandwidth and MCS. Therefore signaling is reduced since there is no need for new UL allocation grants.

For each user $i \in \mathscr{U}$, the scheduler gets the sounded SINR at the PRB level $\gamma_{i, l, r}^{\mathrm{SRS}}$. Hence, it selects the MCS which maximizes the throughput. This is done under the constraint that the estimated block error rate (BLER) is smaller or equal than the target BLER at first transmission. The expected throughput in that $\mathrm{PRB} R_{\mathrm{b}}^{\mathrm{PRB}}(i, j)$ is estimated from the MCS. Finally, a score $s(i, c)$ per cluster $c$ is computed as its average estimated throughput:

$$
s(i, c)=\frac{1}{\left|\mathscr{P}_{c}\right|} \sum_{j \in \mathscr{P}_{c}} R_{\mathrm{b}}^{\mathrm{PRB}}(i, j)
$$

where $\mathscr{P}_{c}$ denotes the set of PRBs in cluster $c$ and $\left|\mathscr{P}_{c}\right|$ is its corresponding cardinality. Here, all clusters have the same size: $\left|\mathscr{P}_{c}\right|=M_{\text {cluster }}$

The result is a matrix $\mathbf{S} \in \mathbb{R}_{+}^{|\mathscr{U}| \times C}$ containing all cluster scores for every user $i \in \mathscr{U}$, ordered following the TD criteria:

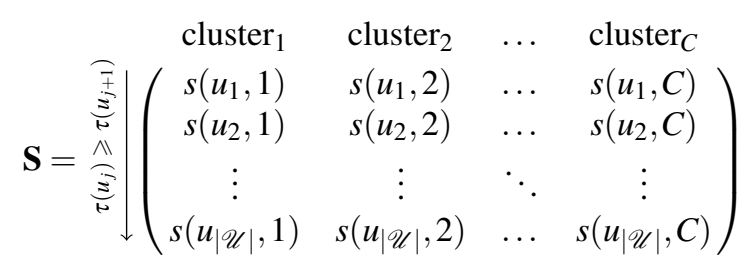

For each user in the sorted list, the FD stage will search the set of clusters of both CCs with the highest score $\mathscr{B}_{i}$. In case more than two clusters share the best value, the scheduler selects those having the lowest accumulated score and so having worse performance in the remaining users.

$$
\begin{gathered}
b_{1}(i)=\min _{c}\left(\sum_{\forall u \in \mathscr{U}} s(u, c)\right), c \in \mathscr{B}_{i} \\
b_{2}(i)=\min _{c}\left(\sum_{\forall u \in \mathscr{U}} s(u, c)\right), c \in \mathscr{B}_{i}-\left\{b_{1}(i)\right\}
\end{gathered}
$$


Next, the scheduler estimates whether power de-rating is going to be applied by the UE and the corresponding impact on its final throughput. This is done by computing the transmission power to be used by the UE. Since SRSs use the same OLPC algorithm as data, the eNode-B just requires to know $P_{\mathrm{SRS}}$. Even though this information is not directly reported, the UE can indicate its current power headroom at the medium access control (MAC) layer. Then, as the eNode-B knows the UE power class (and so its maximum power), the actual power estimation is done. Note that SRS and data might have different allocated bandwidths, but the power spectral density measured at the PRB level $\delta_{\mathrm{SRS}}$ and $\delta_{\mathrm{PUSCH}}$ is kept as long as power levels are below $P_{\max }-\pi_{A}$. Otherwise, the scheduler computes the user MPR from equation 1 or equation 2, depending on the allocated configuration, and updates the power spectral density by subtracting the difference $\Delta P$ from equation 12 .

Given the estimation of transmission power and interference plus noise power (addition at subcarrier level in the allocated clusters), the effective $\gamma_{i, l}^{\mathrm{PUSCH}}$ is obtained and used for link adaptation.

The corresponding throughput is compared against a contiguous allocation. The configuration resulting in a higher throughput is the allocated one.

\section{Simulation Conditions}

To assess the performance of uplink CA operation considering MPR wise scheduling decisions, a dynamic system level simulator has been implemented. The simulation tool is fully programmed in C\#.NET framework.

Realistic long and short term fading is considered. Spatially correlated log-normal variations are introduced, based on the two dimensional correlated shadowing model presented in [33]. An extended typical urban power delay profile is assumed considering a UE speed of $3 \mathrm{~km} / \mathrm{h}$ based on the guidelines of [34].

Antennas are placed at a height of $25 \mathrm{~m}$ and radiation patterns from commercial antennas [35] have been used with downtilt angles optimized for each ISD [36].

The simulation tool includes all the main radio resource management functionalities identified in the UL:

- Channel State Information Manager: It is in charge of allocating the SRS bandwidth. It also collects the sounding signals to compute the sounded SINR.

- Link Adaptation Unit: It selects the suitable MCS based on the sounded SINR. Sounding information is considered to be outdated after 10 TTIs. If there is no available CSI for one particular PRB this is not considered as a possible resource to be allocated.

- Packet Scheduler: This unit runs the different scheduling options previously mentioned. It is also in charge of running the HARQ controller at the eNB side.

All simulation assumptions are summarized in Table I. The simulated scenario has 14 tri-sectorial sites, and 42 eNBs. The wireless access network is considered to have a RTT of $8 \mathrm{~ms}$. Finite buffer communications are assumed and, as soon as the buffer is entirely transmitted, the UE is automatically reconnected in another position. This keeps a constant number of interference sources during the simulation time. The 
Table 2 Simulation scenario assumptions

\begin{tabular}{|c|c|}
\hline Parameter & Value \\
\hline \hline Carrier frequency & $2 \mathrm{GHz}$ \\
\hline Inter-site distance & $500 \mathrm{~m}$ \\
\hline Bandwidth & $20 \mathrm{MHz}$ \\
\hline Power delay profile & Extended Typical Urban \\
\hline Doppler model & Young and Beaulieu [34] 3 km/h \\
\hline Shadowing correlation distance & $50 \mathrm{~m}$ \\
\hline Shadowing deviation & $6 \mathrm{~dB}[37]$ \\
\hline Number of users per cell & 30 \\
\hline Number of scheduled users per TTI & $10[38]$ \\
\hline UE Buffer size & $1 \mathrm{Mb}$ \\
\hline Round trip time & $8 \mathrm{~ms}$ \\
\hline Target BLER & $10 \%$ \\
\hline Adaptive MCS & $16 \mathrm{PRB}$ \\
\hline SRS BW & $2 \mathrm{TTI}$ \\
\hline SRS periodicity & $10 \mathrm{TTI}$ \\
\hline SRS information expiration & $23 \mathrm{dBm}$ \\
\hline Maximum UE transmission power &
\end{tabular}

adjustment of power control parameters largely follows the work in [27], and they are equal in all cells.

Several case studies have been run and compared against several benchmarks:

- Cont: Contiguous SC-FDMA allocation.

- MC: Multi-cluster MPR agnostic allocation. All UEs make use of two separated clusters and CA transmission.

- MC-Th: Threshold algorithm presented in [19] and applied for CA joint scheduling. In this case, the average value of MPR is set to $6 \mathrm{~dB}$.

- MC-MPR: The actual proposal, multi-cluster MPR aware allocation.

\section{Results}

With the aim of analysing the MPR performance under diverse transmission conditions all four algorithms are simulated varying the number of allocated PRBs, and in two different scenarios: an interference limited (ISD $500 \mathrm{~m}$ ) and a noise limited scenario (ISD $1732 \mathrm{~m}$ ) one.

\subsection{Algorithm analysis}

To evaluate the algorithm performance, results are first analyzed in the interference limited scenario with a constant cluster size. Figure 3 shows the cumulative distributive function $(\mathrm{CDF})$ of the average user throughput. Transmitting in more than one 


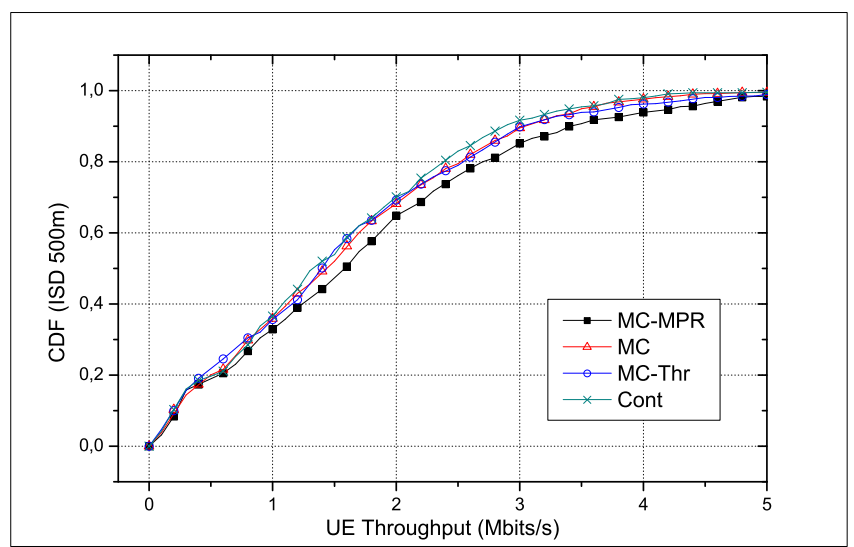

Fig. 3 Average UE Throughput for all simulated strategies. Cluster size $=8$ PRBs.SRS Bandwidth $=16$ PRBs

Table 3 Throughput values for Cluster size $=8$ PRBs

\begin{tabular}{|c|c|c|c|c|}
\hline Throughput & MC-MPR & MC & MC-Thr & Cont \\
\hline \hline Average (Mbits/s) & 1.78 & 1.60 & 1.61 & 1.54 \\
\hline Cell-edge (kbps) & 141 & 121 & 105 & 108 \\
\hline BLER (\%) & 29.24 & 31.54 & 29.27 & 33 \\
\hline
\end{tabular}

cluster of PRBs improves the system performance. It can be seen that in all three MC solutions, the throughput is increased with respect to the contiguous allocation policy. This gain is brought by the extra frequency diversity, since the scheduler enjoys more flexibility to choose the best spectrum areas. Table 7 summarizes the average and cell edge throughput and the resulting BLER obtained at the first attempt for all four cases.

The proposed algorithm lets increase both the average and the cell edge throughput. The MC-MPR solution brings the system into a hybrid solution, in which information about UE's power availability is smartly used by the scheduler to allocate clusters in a contiguous or separated manner. For this particular case the increase in average throughput is higher than $11 \%$ with respect to the case in which all UEs transmit in separated clusters (MC scheduler), and more than 16\% in the 5th percentile worst throughput. If power limited UEs transmit in separated clusters their maximum transmitted power is reduced (affected by the MPR), which has a direct impact on the power spectral density. The improvement seen in the MC-MPR scheduler is because it allows for localized transmissions in power limited cases. Hence, UEs do not require power de-rating and their link budget is not impaired, thus there is no reduction of PSD.

Improvements are also noticeable with respect to the threshold-based scheduler that selects CA users based on their attenuation to the eNB. In this case the average rate increase is similar to the previous strategy, around 11\%; however cell edge throughput increases $34 \%$. MC-Thr solution treats all cell edge UEs equal as they are 


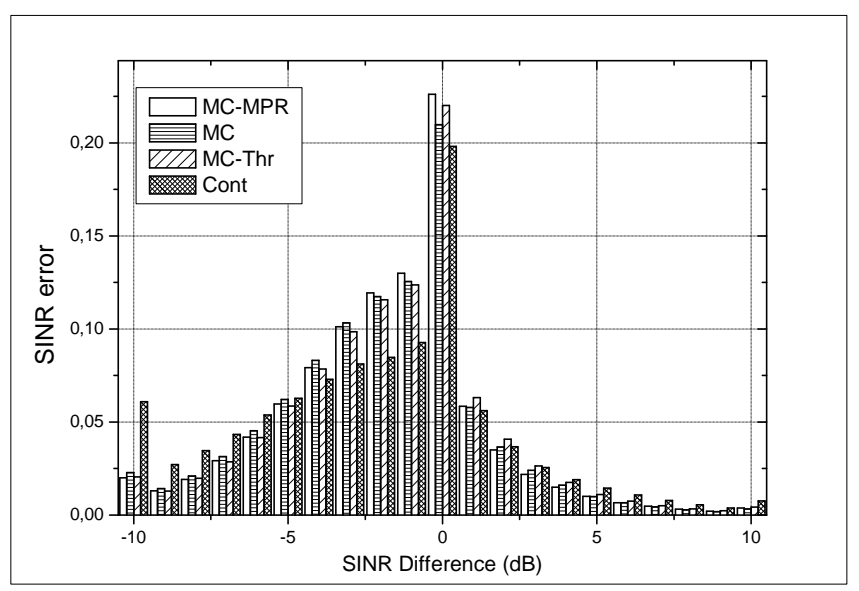

Fig. 4 SINR misalignment for all simulated strategies. Cluster size $=8$ PRBs. SRS Bandwidth $=16$ PRBs

classified given their attenuation with respect to the serving eNB. For a low bandwidth allocation not all UEs in the cell edge are power limited and the gains brought by non-contiguous allocation can improve the 5th percentile worst throughput as well. But, even though UEs are power limited and a separated allocation would imply a PSD reduction the gain brought by frequency diversity overcomes this loss.

As seen in table 3 the solution that provides the lowest BLER at the first attempt is MC-MPR. By definition, the increase in BLER is basically because the average $\overline{\gamma_{i}^{\mathrm{SRS}}}$ differs from the received $\bar{\gamma}_{i}^{\mathrm{PUSCH}}$; Figure 4 shows the difference between both SINR metrics. Apart from the strong interference (and so SINR) variability the UL experiences, applying MPR and reducing the PSD also creates differences between both SINRs. When comparing the BLER improvement in all CA scheduling strategies, the highest improvement is with respect to MC strategy which does never consider $\Delta P$ (if any) in the MCS allocation process. There is no gain with respect to MCThr because in this case, cell edge UEs who may have $\Delta P \neq 0$ are banned from multi cluster transmission, and therefore there is no misalignment between $\gamma_{\mathrm{SRS}}$ and $\gamma_{\mathrm{PUSCH}}$ caused by this factor. As explained in the system model section, the total equivalent $\gamma_{\mathrm{SRS}}$ is calculated as the average of individual $\gamma_{\mathrm{SRS}}^{\mathrm{PRB}}$. For a given coherence band, the channel variation over a lower number of consecutive PRBs tends to be flatter than in a larger allocation. Thus, although scheduling decisions are opportunistic, large localized allocations are more likely to be affected by deep fadings. For this reason the probability of having short term fadings in the allocated region is higher because the correlation among the different PRBs is lower when both are fairly distant. For this reason there is a higher improvement of the BLER with respect to the contiguous bandwidth allocation strategy. Occasional allocation of contiguous resources is allowed in MC-MPR and MC-Thr schedulers. However, in both cases the probability of this event to occur is low.

Figure 5 shows the CDF of the HARQ process. When the SINR difference is high there is an increased probability of having retransmissions, and also of having lost 


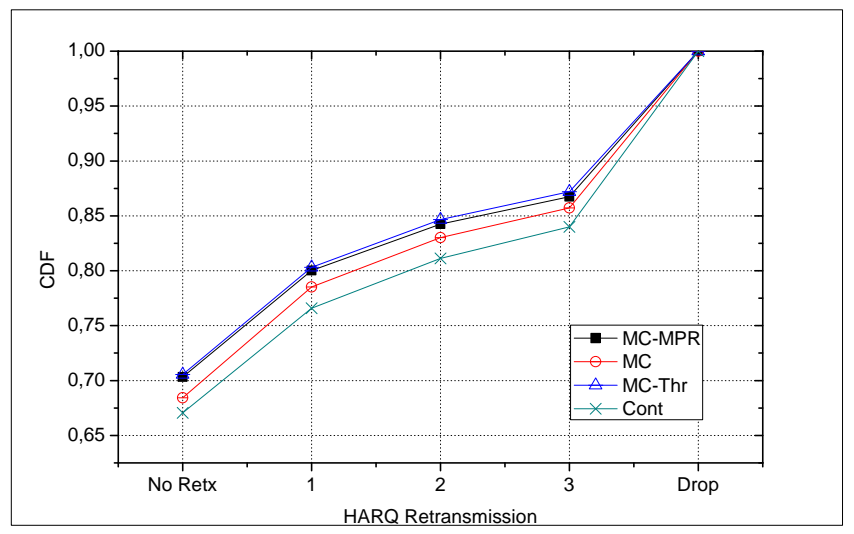

Fig. $5 \mathrm{CDF}$ of the HARQ process - Probability of retransmissions and dropped packets. Cluster size $=8$ PRBs. SRS Bandwidth $=16$ PRBs

packets. High number of retransmissions increases the delay, and therefore reduces the UE throughput.

\subsection{Cluster size impact}

An increase in $M_{\text {Alloc }}$ implies a higher $P_{\mathrm{TX}}$. Therefore, transmitting in separated clusters can severely decrease the PSD as there are more power limited UEs. This implies that for an increase in bandwidth the probability of transmitting in separated clusters must decrease in order to always keep the best performance. Figure 6 shows two metrics that are very much related one to another. First, the probability of transmitting in separated clusters and second the average transmitted power, both versus the cluster size. The threshold based strategy does not consider the allocated bandwidth, therefore as the cluster size increases, the probability of non contiguous allocation remains equal. The optimal selection of CA users is not only related to the path-loss $(L)$ but also to $M_{\text {Alloc }}$ because both control the resulting $P_{\mathrm{TX}}$.

Tables 4 and 5 show the results for increased bandwidth configurations. There is still improvement in throughput for cluster size equal to 10 PRBs, but this improvement is not as significant as it was with 8 PRBs and it is much lower in the case of 20 PRBs. As $M_{\text {Alloc }}$ grows, the gain brought by multi-clustered transmissions reduces with respect to contiguous ones. This is because the increase in UE power demand to transmit such a high bandwidth overcomes the multi-clustering gain seen in lower bandwidth configurations.

\subsection{Performance under large ISDs}

In a noise limited scenario, with ISD $=1732 \mathrm{~m}, \overline{P_{\mathrm{TX}}}$ increases due to the high pathloss to the eNB. The cluster size must be kept low, to assure that a low number of UEs transmit under maximum power conditions. In the last section it has been seen that 


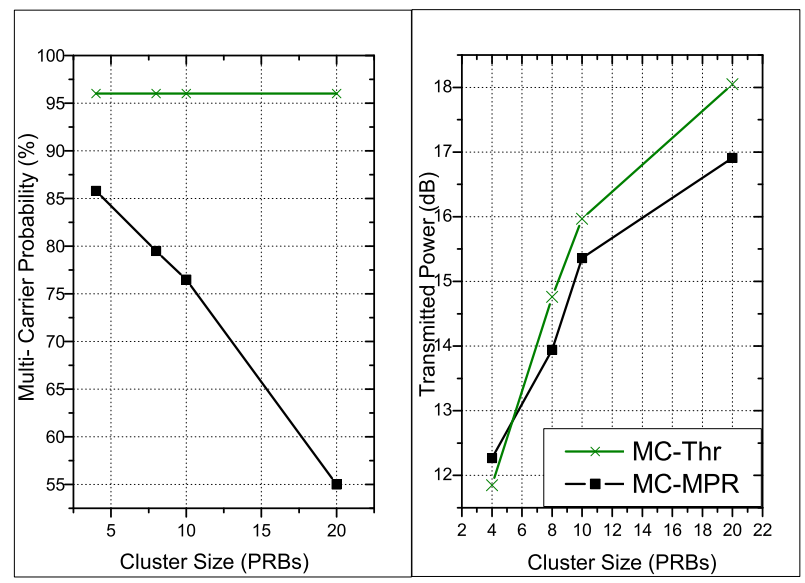

Fig. 6 Probability of transmitting in separated clusters vs Transmitted power

Table 4 Throughput values for Cluster size $=10$ PRBs

\begin{tabular}{|c|c|c|c|c|}
\hline Throughput & MC-MPR & MC & MC-Thr & Cont \\
\hline \hline Average (MBits/s) & 2 & 1.9 & 1.88 & 1.7 \\
\hline Cell-edge (kbps) & 131 & 121 & 127 & 102 \\
\hline BLER $(\%)$ & 28 & 28.6 & 28 & 31 \\
\hline
\end{tabular}

Table 5 Throughput values for Cluster size $=20 \mathrm{PRBs}$

\begin{tabular}{|c|c|c|c|c|}
\hline Throughput & MC-MPR & MC & MC-Thr & Cont \\
\hline \hline Average (MBits/s) & 2.86 & 2.85 & 2.85 & 2.7 \\
\hline Cell-edge (kbps) & 131 & 123 & 120 & 137 \\
\hline BLER (\%) & 27 & 27 & 27 & 28 \\
\hline
\end{tabular}

the probability of transmitting in separated clusters is closely related to the average transmitted power. In a large ISD condition, this probability versus the transmitted power is shown in table 6 .

The $\overline{P_{\mathrm{TX}}}$ under noise limited conditions is close to the one in the interference limited scenario with a cluster size of $20 \mathrm{PRBs}$, near $17 \mathrm{dBm}$. When comparing the probability of transmitting in separated clusters of both scenarios, the noise limited has an increase of $20 \%$ with respect the interference limited one. This is because both transmission power distributions are fairly different as shown in Figure 7. Although average power values are similar, the probability of having one user transmitting at maximum power levels is still larger for the interference limited scenario with higher transmission bandwidth. Therefore the result is that in large ISDs the algorithm is able to successfully allocate more UEs in separated clusters.

As the path-loss to the eNB increases with higher ISDs, the MC-Thr algorithm decreases the probability of transmitting in separated clusters with respect to the lower 
Table 6 Probability of transmitting in separated clusters vs Transmitted power for Cluster size $=4$ PRBs

\begin{tabular}{|c|c|c|}
\hline & MC-MPR & MC-Thr \\
\hline \hline $\mathrm{P}(\mathrm{CA})(\%)$ & 75 & 65 \\
\hline$\overline{P_{\mathrm{TX}}}(\mathrm{dBm})$ & 17 & 17.8 \\
\hline
\end{tabular}

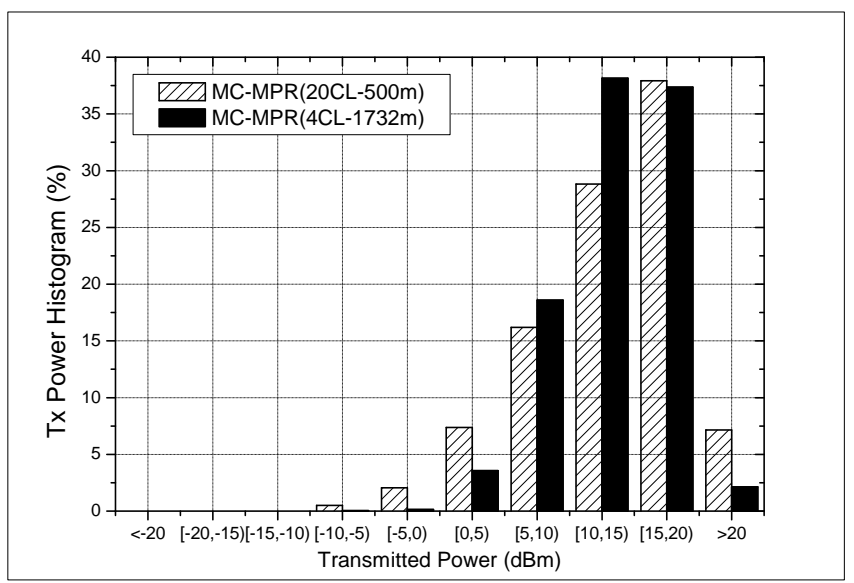

Fig. 7 Transmitted power probability density function comparison between interference and noise limited scenarios

Table 7 Throughput values for Cluster size $=4$ PRBs

\begin{tabular}{|c|c|c|c|c|}
\hline Throughput & MC-MPR & MC & MC-Thr & Cont \\
\hline \hline Average (kbits/s) & 472 & 463 & 423 & 409 \\
\hline Cell-edge (kbits/s) & 61 & 45 & 66 & 53 \\
\hline BLER (\%) & 34 & 36 & 34 & 35 \\
\hline
\end{tabular}

ISD case. However, as seen in table 6 there is still a $10 \%$ difference when comparing it to the proposed algorithm.

Table 7 summarizes the throughput results. The increase in distance to the serving cell results in an overall throughput reduction, when compared to the low ISD performance. Throughput gains when $L$ grows are similar to those with increased bandwidth. The improvement of MPR wise decisions in opportunistic scheduling can still be appreciated with a lower percentage of gain. It is important to notice how much the cell edge performance is impaired due to forced multi-clustered transmissions; there is a $35 \%$ difference in the cell edge with respect to the MC strategy. However, it is still beneficial to allow opportunistic non-contiguous allocations under high path-loss circumstances, given that cell-edge gains are still appreciated. 


\section{Conclusion}

A novel scheduling algorithm has been presented in which the effect of the MPR is taken into account. The estimated user channel information obtained via SRS is employed to assess the gain/loss of the cluster allocation on each specific case. No new signalling operations are required to run this algorithm.

Four scheduling algorithms have been compared: Contiguous, multi-cluster, multicluster based on a path-loss threshold and MPR based scheduling. Results show that the proposed strategy overcomes all these benchmarks. Adding the MPR information and assessing the UE capability of transmitting in separated clusters of PRBs lets the system improve the overall performance. It has been shown that the cell edge and average UE capacity are improved when considering individual gains of CA transmission. MPR wise decisions reduce the misalignment between the CSI obtained through the SRSs and the SINR in the shared channel. This yields to an improved BLER and a the corresponding reduction in retransmissions and discarded packets.

The potential gains of multi-cluster transmission are shown to depend on the power increases due to (a) larger bandwidth allocations and (b) the increase in pathloss due to larger ISD. In the first case, is the only solution that reacts and adjusts the use of clustered transmissions to the new power conditions. On the other hand, the probability of transmitting in a non-localized manner remains constant in the MCThr case. The performance under large ISDs is still improved, allowing opportunistic non-contiguous transmissions in the cell edge when the allocation is profitable to the UE.

In low ISD scenarios with short bandwidth allocations, non-contiguous transmissions are preferred against classical uplink localized carrier mapping. When UEs transmit below the maximum available levels, UEs can improve their spectral efficiencies by transmitting in separated pieces of spectrum. However, as the transmission power increases, contiguous allocation of PRBs in power limited UEs can improve its performance. Based on this, MC-MPR analyses each UE case, coming up with the solution that best fits the system performance. In this sense, the solution adapts itself to different scenarios, resulting in a better performance.

As a final remark, SRSs are crucial for the correct functioning of the algorithm as they provide all the information for the channel estimation. Therefore, the SRSs allocation is an important parameter that must be fine tuned in order to obtain the best performance of the algorithm. Future work should go in that direction.

Acknowledgements This work is supported by the Spanish National Science Council through the project TEC2011-27723-C02-01 and by the European Regional Development Fund (ERDF).

\section{References}

1. D. Martín-Sacristán, J.F. Monserrat, J. Cabrejas-Peñuelas, D. Calabuig, S. Garrigas, and N. Cardona. On the way towards fourth generation mobile: 3gpp lte and lte-advanced. EURASIP Journal on Wireless Communications and Networking, 2009:4, 2009. 
2. Eiko Seidel. 3gpp lte-a standardization in release 12 and beyond. Nomor Research GmbH, Munich, Germany, 2013.

3. 4G-Americas. 4G Mobile Broadband Evolution-Rel 10 Rel 11 and Beyond . http://www . 4gamericas .org.

4. Gilberto Berardinelli, TB Sorensen, Preben Mogensen, and Kari Pajukoski. Transmission over Multiple Component Carriers in LTE-A Uplink. Wireless Communications, IEEE, 18(4):67-73, 2011.

5. 3GPP. System Level Performances of Non-contiguous RB Assignment for LTEAdvanced Uplink. Technical Report R1-090020, 3GPP TSG-RAN, January 2008.

6. 3GPP. Evolved Universal Terrestrial Radio Access (E-UTRA); Long Term Evolution (LTE) physical layer; General description. TS 36.201, 3rd Generation Partnership Project (3GPP), December 2007. URL http://www. 3gpp.org/ftp/Specs/html-info/36201.htm.

7. 3GPP. PAPR of UL Access Schemes. Technical Report R1-083780, 3GPP TSGRAN, October 2008.

8. 3GPP. User Equipment (UE) Radio Transmission and Reception (Release 11). TS 36.101, 3rd Generation Partnership Project (3GPP), September 2012. URL http://www . 3gpp.org/ftp/Specs/html-info/36101.htm.

9. Yuanye Wang, K.I. Pedersen, P.E. Mogensen, and T.B. Srensen. Resource Allocation Considerations for Multi-Carrier LTE-Advanced Systems Operating in Backward Compatible Mode. In Personal, Indoor and Mobile Radio Communications, 2009 IEEE 20th International Symposium on, pages 370 -374, sept. 2009. doi: 10.1109/PIMRC.2009.5450150.

10. Yuanye Wang, K.I. Pedersen, T.B. S andrensen, and P.E. Mogensen. Carrier Load Balancing and Packet Scheduling for Multi-Carrier Systems. Wireless Communications, IEEE Transactions on, 9(5):1780 -1789, may 2010. ISSN 1536-1276. doi: 10.1109/TWC.2010.05.091310.

11. Liu Liu, Mingju Li, Juejia Zhou, Xiaoming She, Lan Chen, Y. Sagae, and M. Iwamura. Component Carrier Management for Carrier Aggregation in LTEAdvanced System. In Vehicular Technology Conference (VTC Spring), 2011 IEEE 73rd, pages 1 -6, may 2011. doi: 10.1109/VETECS.2011.5956228.

12. Yuanye Wang, K.I. Pedersen, T.B. Sorensen, and P.E. Mogensen. Utility Maximization in LTE-Advanced Systems with Carrier Aggregation. In Vehicular Technology Conference (VTC Spring), 2011 IEEE 73rd, pages 1-5, may 2011. doi: 10.1109/VETECS.2011.5956494.

13. F. Liu, K. Zheng, W. Xiang, and H. Zhao. Design and Performance Analysis of An Energy-Efficient Uplink Carrier Aggregation Scheme. Selected Areas in Communications, IEEE Journal on, 32(2):197-207, February 2014. ISSN 07338716. doi: 10.1109/JSAC.2014.141202.

14. T. Iwai, A. Matsumoto, S. Takaoka, Y. Ogawa, A. Nishio, and D. Imamura. System Performance of Clustered DFT-S-OFDM Considering Maximum Allowable Transmit Power. In Communications (ICC), 2011 IEEE International Conference on, pages 1 -5, june 2011. doi: 10.1109/icc.2011.5963492.

15. Hua Wang, C. Rosa, and K. Pedersen. Performance Analysis of Downlink Inter-Band Carrier Aggregation in LTE-Advanced. In Vehicular Tech- 
nology Conference (VTC Fall), 2011 IEEE, pages 1 -5, sept. 2011. doi: 10.1109/VETECF.2011.6092836.

16. Hui Tian, Songtao Gao, Jianchi Zhu, and Lan Chen. Improved Component Carrier Selection Method for Non-Continuous Carrier Aggregation in LTEAdvanced Systems. In Vehicular Technology Conference (VTC Fall), 2011 IEEE, pages 1 -5, sept. 2011. doi: 10.1109/VETECF.2011.6092963.

17. Chunyan Li, Ben Wang, Weidong Wang, Yinghai Zhang, and Xinyue Chang. Component carrier selection for LTE-A systems in diverse coverage carrier aggregation scenario. In Personal Indoor and Mobile Radio Communications (PIMRC), 2012 IEEE 23rd International Symposium on, pages 1004-1008. IEEE, 2012.

18. Zhao Ji-hong, Li Hui, and Qu Hua. A SPF-PF crossing Component Carrier joint scheduling algorithm. In Advanced Communication Technology (ICACT), 2012 14th International Conference on, pages 173-177, 2012.

19. Hua Wang, C. Rosa, and K. Pedersen. Uplink Component Carrier Selection for LTE-Advanced Systems with Carrier Aggregation. In Communications (ICC), 2011 IEEE International Conference on, pages 1 -5, june 2011. doi: 10.1109/icc.2011.5963279.

20. Hua Wang, Hung Nguyen, C. Rosa, and K. Pedersen. Uplink Multi-cluster Scheduling with MU-MIMO for LTE-Advanced with Carrier Aggregation. In Wireless Communications and Networking Conference (WCNC), 2012 IEEE, pages 1202 -1206, april 2012. doi: 10.1109/WCNC.2012.6213960.

21. Rajarajan Sivaraj, Amit Pande, Kai Zeng, Kannan Govindan, and Prasant Mohapatra. Edge-Prioritized Channel and Traffic-Aware Uplink Carrier Aggregation in LTE-Advanced Systems. In World of Wireless, Mobile and Multimedia Networks (WoWMoM), 2012 IEEE International Symposium on a, pages 1-9. IEEE, 2012.

22. Hua Wang, C. Rosa, and K. Pedersen. Performance of Uplink Carrier Aggregation in LTE-Advanced Systems. In Vehicular Technology Conference Fall (VTC 2010-Fall), 2010 IEEE 72nd, pages 1 -5, sept. 2010. doi: 10.1109/VETECF.2010.5594543.

23. G. Berardinelli, T.B. Sorensen, P. Mogensen, and K. Pajukoski. Transmission over Multiple Component Carriers in LTE-A Uplink. Wireless Communications, IEEE, 18(4):67 -73, august 2011. ISSN 1536-1284. doi: 10.1109/MWC.2011.5999766.

24. 3GPP. MPR for LTE Multi Cluster Transmission. Technical Report R4-110955, 3GPP TSG-RAN, February 2011.

25. 3GPP. MPR for Non-contiguous Allocation. Technical Report R4-110265, 3GPP TSG-RAN, January 2011.

26. 3GPP. LTE-A MC RF Requirements for Contiguous Carriers. Technical Report R4-091910, 3GPP TSG-RAN, May 2009.

27. M. Lema, M. Garcia, J.J. Olmos, and S. Ruiz. On the Performance LTE UL Power Control in Realistic Conditions. 4th International Conference on Mobile Networks and Management, 2012. ICTS, september 2012.

28. C.U. Castellanos, D.L. Villa, C. Rosa, K.I. Pedersen, F.D. Calabrese, P.H. Michaelsen, and J. Michel. Performance of Uplink Fractional Power Control 
in UTRAN LTE. In Vehicular Technology Conference, 2008. VTC Spring 2008. IEEE, pages 2517-2521. IEEE, 2008.

29. 3GPP. PUSCH Power Control for LTE-Advanced. Technical Report R1-092574, 3GPP TSG-RAN, July 2009.

30. 3GPP. Physical Layer Procedures. TS 36.213, 3rd Generation Partnership Project (3GPP), September 2009. URL http://www . 3gpp.org/ftp/Specs/html-info/36213.htm.

31. Holma. $\mathrm{H}$ and A. Toskala. LTE-A 3GPP Solution for IMT-Advanced. Wiley Online Library, 2012.

32. A. Pokhariyal, K.I. Pedersen, G. Monghal, I.Z. Kovacs, C. Rosa, T.E. Kolding, and P.E. Mogensen. HARQ Aware Frequency Domain Packet Scheduler with Different Degrees of Fairness for the UTRAN Long Term Evolution. In Vehicular Technology Conference, 2007. VTC2007-Spring. IEEE 65th, pages 2761 -2765, april 2007. doi: 10.1109/VETECS.2007.567.

33. R. Fraile, O. Lazaro, and N. Cardona. Two Dimensional Shadowing Model. Tr available as $\operatorname{td}(03) 171, \operatorname{COST} 273,2003$.

34. D.J. Young and N.C. Beaulieu. The Generation of Correlated Rayleigh Random Variates by Inverse Discrete Fourier Transform. Communications, IEEE Transactions on, 48(7):1114 -1127, jul 2000. ISSN 0090-6778. doi: 10.1109/26.855519.

35. Kathrein Website. http://www.kathrein.com/en/.

36. M. Garcia-Lozano and S. Ruiz. Effects of Downtilting on RRM Parameters. In 15th IEEE International Symposium on Personal, Indoor and Mobile Radio Communications, 2004. PIMRC 2004., volume 3, pages 2166-2170 Vol.3, 2004. doi: 10.1109/PIMRC.2004.1368381.

37. 3GPP. Further Advancements for E-UTRA; Physical Layer Aspects. TS 36.814, 3rd Generation Partnership Project (3GPP), March 2010. URL http://www.3gpp.org/ftp/Specs/html-info/36814.htm.

38. Francesco Capozzi, Daniela Laselva, Frank Frederiksen, Jeroen Wigard, Istvan Kovacs, and Preben Mogensen. Utran lte downlink system performance under realistic control channel constraints. In IEEE 70th Vehicular Technology Conference Fall (VTC 2009-Fall), 2009. IEEE, 2009. ISBN 978-1-4244-2515-0. doi: 10.1109/VETECF.2009.5378831. 